\title{
Bipolar affective disorder: A review of novel forms of therapy
}

\author{
Ewelina Dziwota ${ }^{1}$, Barbara Drapala ${ }^{2}$, Magdalena Gaj ${ }^{2}$, \\ Nikodem SKoczen ${ }^{1}$, Marcin Olajossy ${ }^{1}$
}

${ }^{1}$ II Department of Psychiatry and Psychiatry Rehabilitation, Gluska 2, 20-439 Lublin, Poland

${ }^{2}$ Medical Students' Research Association of the Medical University of Lublin, Poland

\begin{tabular}{l}
\hline ARTICLE INFO \\
\hline Received 09 March 2015 \\
Accepted 28 April 2015
\end{tabular}

Keywords:

allopurinol,

$\mathrm{N}$-acetylcysteine,

memantine,

acetylsalicylic acid, ketamine,

modafinil,

tamoxifen.

\begin{abstract}
Normothymic, antidepressant and antipsychotic pharmaceutics are, in accordance with international guidelines, employed both in the therapy and the prevention of bipolar disorder (BD). Long-term studies on the mechanisms of action of such medications, as well as on the pathogenetic background of $\mathrm{BD}$, have led to the discovery of effective, albeit unconventional pharmacotherapeutic approaches. These methods have the potential to successfully treat mania and depression, as well as to counter affective episode relapse. Allopurinol - commonly used to treat gout, secondary hyperuricemia and Lesch-Nyhan syndrome, acts by inhibiting the synthesis of uric acid, levels of which are often increased in manic patients. Due to this, an evaluation of the potential effect of allopurinol on the reduction of mania symptoms seems to be reasonable. Additionally, the numerable research papers coming out of research regarding the role of purine neurotransmitters in mood alterations, indicate that adenosine agonists act analogously to dopamine antagonists.

$\mathrm{N}$-acetylcysteine (NAC), a supporting agent in the pharmacotherapy of depressive episodes in bipolar disorder, substantially accelerates mood stabilization in patients. In turn, memantine, known for its procognitive effect, not only has antimanic and normothymic properties, but also boosts the neuroprotective action of traditional lithium therapy.

In view of the latest opinions, the subtle pro-inflammatory process is typical for the pathophysiology of bipolar disease. Acetylsalicylic acid (ASA) (a popular analgesic, antipyretic and antiphlogistic agent) may be useful in $\mathrm{BD}$ therapy. This is because that, via its effect upon cyclooxygenase-1 (COX-1) and cyclooxygenase-2 (COX-2), ASA modulates leukocyte recruitment in neuro-inflammation.

Apart from the above-mentioned medications, this article introduces the results of recent investigations of ketamine, modafinil and tamoxifen, including their receptor mechanisms, as well as certain genetic aspects or elements of their pharmacodynamics, for use in $\mathrm{BD}$ therapy. We put forward that, possibly, more insightful cognition of these drugs will allow significant enrichment in the range of pharmacotherapy for BD in the near future.
\end{abstract}

\section{INTRODUCTION}

Normothymic, antidepressant and antipsychotic pharmaceutics, by way of international guidelines, have been approved for employment in both the therapy and prevention of BD. Long-term studies on the mechanisms of action of the above-mentioned medications, as well as studies on the pathogenetic background of $\mathrm{BD}$, have led to the discovery of unconventional, albeit potentially successful pharmacotherapeutic methods, for treating mania and depression or in counteracting affective episode relapse.

This article constitutes a review of the medications: allopurinol, NAC, memantine, ASA, ketamine, modafinil and tamoxifen.

\footnotetext{
* Corresponding author

e-mail: dziwota.e@gmail.com
} 


\section{ALLOPURINOL}

Allopurinol is an organic chemical compound that is an isomer of hypoxanthine. In pharmacotherapy, it acts through the inhibition of xanthine oxidase (XO), and is commonly used to treat e.g. gout, secondary hyperuricemia and LeschNyhan syndrome. Via its mechanism of action, allopurinol reduces synthesis of the uric acid, whose levels, as had been observed, are often elevated in manic patients. Thus, it may cause alleviation of manic symptoms [9]. Over the past few years, there has been a growing number of case reports and clinical trials indicating a correlation between the purine system and BD. However, this is not a complete novelty, as in 1913, Kraepelin noted a relationship between manic symptoms and elevated uric acid level. Moreover, in 1949, the Australian psychiatrist, John Cade, put forward that acute manic episodes may probably be evoked by auto-intoxication with uric acid [9]. He also observed and wrote of the mood-normalizing effects of lithium. There is a growing evidence of the role of purine neurotransmitters disorders in mood alterations, as adenosine agonists most likely act analogously to dopamine antagonists. Research has confirmed the interactions between dopaminergic and adenosine receptors in the striatum. Hence, an increase in adenosine agonist levels results in a decrease of dopaminergic agonist affinity [21]. Through inhibition of XO, allopurinol acts favourably on purine retrieval, increases brain concentrations of endogenous adenosine, and, thus, may hypothetically alleviate manic symptoms [6].

In attempting to understand allopurinol as a potential pharmacotherapeutic, one clinical trial had been designed to demonstrate whether allopurinol therapy is of benefit to patients in the acute manic phase who had been treated with valproic acid [9]. beyond this, another trial had had the goal of evaluating the effects of allopurinol as a supplement to conventional therapy with mood stabilizers or antipsychotics $[21,6]$.

The effects of the above-mentioned trials were more recently assessed by way of using the Young Mania Rating Scale (YMRS). Possible conclusions drawn after analysis, are intriguing. First of all, allopurinol, indeed, leads to more rapid mood stabilization in patients treated with valproate (as compared to placebo) [9]. Secondly, a comparison between allopurinol action and the effects exerted by various mood stabilizers or antipsychotics is not entirely reliable. This is due to the occurrence of obscure and diverse interactions of particular agents with allopurinol. Therefore, a strict contrast between patients treated with allopurinol and those receiving placebo, is not outwardly evident $[21,6]$. Thirdly, it is interesting to note that, despite taking diverse medications, patients who withdrew from caffeine for the time of clinical trials demonstrated better and positive effects, than those on placebo. This is because caffeine acts antagonistically on adenosine receptors and agonistically on dopaminergic receptors, thus its absence results in seeing the undistracted action of the investigated drug [6].

Allopurinol, when all the aspects of its action are considered, may actually accelerate mood stabilization in patients with manic episode. Hence, is worth considering as a treatment option. However, attention should be paid to certain co-administered drugs when allopurinol is considered as a pharmacotherapeutic, as, in a particular patient, possible contraindications, as well as the presence or absence of caffeine addiction, has influence on therapeutic progress.

\section{N-ACETYLCYSTEINE}

An organic chemical compound, $\mathrm{N}$-acetylcysteine is a $\mathrm{N}$-acetyl derivative of L-cysteine. Because of its mucolytic properties, it is often used in the treatment of both acute and chronic bronchitis or bronchiolitis, bronchiectasis, cystic fibrosis, lung emphysema and numerous other airway pathologies characterized by the presence of large amounts of thick mucus or mucopurulent secretions. It is also capable of neutralizing the toxic metabolites of acetaminophen, which is why NAC is used in cases of such poisoning.

There appears newer evidence supporting the thesis which states that numerous psychiatric conditions, including BD, are accompanied by redox reactions dysregulation that may be susceptible to appropriate treatment [13]. Glutathione is the predominant non-enzymatic intracellular neutralizer of free radicals in the brain, as well as the main endogenous antioxidant in view of brain function [4]. This chemical is synthesized from amino acid precursors (L-glutamate, L-cysteine and L-glycine) in two enzymatic reactions. In oxidative stress conditions, glutathione is prone to depletion. Through enlarging the body's resources of L-cysteine (by NAC administration), the ratio of glutathione synthesis to precursor amount is altered, which, in turn, leads to the elevation of glutathione concentration in the brain [4].

The main purpose of the clinical trials which investigated the influence of NAC on the progression of BD, was to ascertain whether the drug modifies the course of major depressive episodes. These are often treated with multiple medications due to lack of response to current mono pharmacotherapy [4]. Two of the trials were double-blind, placebocontrolled studies. All the trials were based on the assumption that supplementary administration of NAC, in addition to current pharmacotherapy, will shorten the time until the induction of normothymic effect within the patients taking part in the study [4].

The research results of such work are essentially similar. As compared to the control group, mood stabilization was achieved faster in patients from within the study group, with the greater or lesser effect depending on the study and assessment scale $[13,4,3]$. What we see is a promising dependence, which in the future may surely bring intriguing concepts into the treatment of depressive episodes in BD [13].

\section{MEMANTINE}

Memantine is an organic chemical compound that is a non-competitive antagonist of the N-Methyl-D-aspartate (NMDA) receptor. By inhibiting the receptors, it influences their constitutive activity as induced by excessive glutamate release. Memantine is used as a procognitive drug - it stimulates the metabolism of the central nervous system, which, 
in turn, should lead to the activation of conscious mental processes - level of consciousness, the memory phenomena etc. Its major application is in the treatment of Alzheimer's disease.

Recently, it has been demonstrated that memantine exerts strong neuroprotective effects. The fact is that excessive glutamate receptor stimulation induces toxicity and is a part of the etiology of various neurodegenerative disorders, including mood alterations, according to the neurotrophic factor concept [10]. Genetic tests and investigation of proteins synthesized from certain genes have revealed the role of NMDA system in the etiology of BD. This has been documented by the research on glutamate brain levels in patients affected by this particular disorder [7]. By blocking pathological extrasynaptic activity, while preserving normal synaptic functions, memantine may be considered to be a neuroprotective factor [10].

It is worth mentioning that similar properties (neurotrophic, neuroprotective) are also listed in in lithium pharmacotherapy. Lithium and memantine, however, act differently. Memantine decreases both toxicity and additional stimulation of the NMDA receptor, while lithium expands the number of pro-surviving particles in cells, as well as lowers the amount of pro-apoptotic substances [10].

Clinical trials focused on memantine provide promising information on its possible application in therapy of BD. One of such studies was focused on the antimanic and mood-stabilizing efficacy of memantine, especially in treatment-resistant types of BD. In such work, it has been demonstrated, that not only has memantine, significant therapeutic effects, it is also an enhancing factor in the potency of conventional therapy (the research included e.g. lithium). In addition, the effectiveness of treatment was long-lasting. The study was conducted within a group of 40 patients who had been under observation for a year. In the work, remarkable progress was noted in $72.5 \%$ of the patients just after 6 months. Moreover, the study underlined the good tolerance and drug use safety of memantine pharmacotherapy [10].

Another research study of the properties of memantine was actually a pre-clinical inquiry. In this work, pathophysiological lesions, resembling those found in $\mathrm{BD}$, were induced on rats, in order to investigate the potential efficacy of memantine. Strophanthin was then used to mimic certain changes observed in consecutive phases of BD. This study and related studies, indicate that memantine appears to reduce long-term strophanthin-induced motoric hyperactivity, along with impeding strophanthin-evoked hippocampal impulse circulation in rats [7]. However, it is incapable of substantially reducing the cyclic transmission of stimuli in the hippocampus [7].

A comparison of the efficacy of lamotrigine monotherapy and lamotrigine plus memantine treatment was the subject of another analysis. The randomized double-blind, placebo-controlled trials were aimed at verifying whether the addition of memantine increases the efficacy of treatment. Primarily, the result of this inquiry was that lamotrigine, an anticonvulsant agent which decreases presynaptic glutamate levels, exerts certain effects in BD. Nonetheless, pharmacotherapy trials with lamotrigine and lamotrigine plus memantine demonstrated that such treatment is successful only in $40-50 \%$ of all patients. Such work, however, suggests that memantine enhancement does generate an early antidepressant effect, but some more extensive studies are required [2].

\section{ACETYLSALICYLIC ACID}

Acetylsalicylic acid is an organic chemical compound, an acetyl derivative of salicylic acid. It is an irreversible COX-inhibitor acting through the acylation of the catalytic centre, which, in turn, leads to the inhibition of e.g. prostaglandin and prostacyclin formation. In this way, ASA exerts analgesic, antipyretic and antiphlogistic effects, and, for this reason, it is classified as a nonsteroidal anti-inflammatory drug. In appropriately low doses, ASA acts as an antithrombotic agent, by blocking the intrathrombocytic COX-enzyme responsible for thromboxane synthesis - thus, decreasing platelet aggregation potential.

Inflammation is a component of the non-specific immune response to trauma, tissue damage or microorganism invasion. On one hand, this inflammatory process is inevitable for survival, but on the other, an excessive or ill-aimed reaction may lead to pathology or disease - e.g. allergy [20].

Recent clinical studies have shown that a subtle inflammatory process is characteristic for the pathophysiology of BD. The progressive deterioration of certain cognitive functions, consequently reported in $\mathrm{BD}$, confirms the potential role of neuro-inflammation in this disorder [20]. Such work indicate that inflammation may play a role both in origination and reduction of the amount of synapses or neurons, leaving only the most efficient configurations [20]. This action is thought to be exerted by inflammatory influences upon the microglial cells (which are responsible for the remodelling of the dendrites and the loss of the glia or neurons) [17]. It is worth mentioning, that post-mortem studies on patients affected by BD, have revealed elevated levels of arachidonic acid cascade markers [19].

Neuro-inflammation is often associated with the above mentioned synaptic damage and neural loss, both of which may be triggered by factors such as disease, stress, hypoxia or the cell damage evoked by e.g. neurotoxic exposure [20].

Various clinical trials have been conducted on the inflammatory process being a possible cause of BD. Their main aim was conventional therapy enhancement, or even substitution with different medications, especially in treatmentresistant forms of the disorder [20,17,19]. Such work has demonstrated that, because of its effects on COX-1 and COX-2, ASA acts as a modulator of leukocyte recruitment during neuro-inflammation events $[20,17]$.

One of such studies consisted of pre-clinical research on rats. In this study, lithium and other mood stabilizers were assessed as possible anti-inflammatory agents effective in the nervous system, the theory being that they were supposed to decrease the formation of arachidonic acid metabolites, and, thus, to reduce symptoms. As the previously mentioned medications are not efficient in some patients (possibly due to genetic reasons), other drugs altering neuro-inflammation events are worth investigating [19]. 


\section{KETAMINE}

This drug is employed, both in human medicine and veterinary medicine, for pre-operative anesthesia. The pharmacological action of ketamine is mainly based on antagonization of the NMDA receptor. Despite inducing narcosis, ketamine also exhibits analgesic properties. It is, thus, utilized as a precursor to complex anesthesia, and is rarely employed in anesthesiology as an independent anesthetic agent. Ketamine, as mono-anesthetic, is, however, well-suited for short procedures which do not evoke visceral pain. Hence, is is administered for certain diagnostic procedures in children, for plastic surgery, as well as for alleviating the effects of painful changes of burn dressings. It is also employed in short-term surgical procedures.

As shown in certain studies, the glutamatergic system has a significant role in the mechanism of action of agents exhibiting antidepressant efficiency. These medications exert influence upon the NMDA receptor, and e.g. bring about a decrease in NR-1 homologue levels within the brain cortex and the subcortical structures, together with lessening the messenger Ribonucleic Acid (mRNA) expression of the MR2A and NR2B receptor subunits [16]. Such an effect is achieved by way of administering ketamine - a NMDA receptor antagonist [8]. Furthermore, it has been demonstrated that ketamine pharmacotherapy instantly reduces the depressive symptoms in patients who have withdrawn into the depressive phase of BD [8]. Ketamine is also a partial agonist affecting the $\mathrm{D} 2$ dopaminergic receptor, which plays a role in pathophysiology of $\mathrm{BD}$, principally in depressive episodes. As an agonist, ketamine reduces symptoms such as, e.g. anhedonia [12].

Ketamine - dedicated studies mainly concentrate on the antidepressant properties of this agent. Some particular trials involving the drug have been aimed at alleviating symptoms in patients who exhibit resistance to conventional treatment, as well at aiding patients suffering from bipolar disorder with the anxiety component [8]. It has to be stressed that patients with anxiety not only present worse outcomes, but also are more difficult to treat by way of using traditional medicines than are patients with BD that is with a lack of or with a low intensity of anxiety.

One of such studies included 42 patients, all of whom had been previously treated with conventional therapy. In this work, each of the patients received a single intravenous infusion of ketamine administered over 40 minutes. The subject of investigation was to determine the magnitude of change in the manifestation of clinical symptoms in all the patients. The results, however, were quite unambiguous, yet, the average depression severity (assessed using the Hamilton Rating Scale for Depression (HDRS)) was found to be substantially lower, and it must be emphasized, that only a single infusion of ketamine had been used [16]. Subsequent research focused on patients with a treatmentresistant type of BD.

In a further study, the researched group was comprised of 36 patients with bipolar disorder, either with or without highly intense anxiety. The outcome of this work was that a noticeable reduction in symptom severity has been observed in all of the patients after a single administration of ketamine. In this study. The HDRS scale was also used to estimate the severity of depression [8].

Another study assessed the anti-anhedonic effect of ketamine administration. The efficacy of ketamine was determined through the use of brain positron emission tomography (PET). In the study, 36 patients were qualified for the trial. As a result of this work, it was seen that ketamine pharmacotherapy significantly reduced anhedonia, especially in comparison with a placebo. The effect of ketamine administration was clearly visible in image studies, where differences that occurred in glutamatergic transmission were notably evident [12].

\section{MODAFINIL}

As there are a few types of Bipolar disorders evidenced, adequate treatment relies on the proper diagnosis of $\mathrm{BD}$ type with a particular patient. Numerous pharmacotherapy options are available, yet all of these have limitations. Because of this, certain cases of BD are resistant to standard treatment, e.g. normothymics, antipsychotic agents, polytherapy, etc. [5]. What is more, depressive symptoms are often drug-resistant. Oftentimes, they require antidepressive medication administration, nonetheless, some patients do not respond to such therapy. Of note: the efficacy of these agents arouses much controversy (apart from inefficiency) because of possibly increased suicidal risks, excessive mood elevation, and acceleration of cyclic mood changes $[5,18]$. Among the new agents employed in the adjuvant therapy of $\mathrm{BD}$, dopaminergic neurotransmission modifiers are the most promising, especially as far as depressive episodes are concerned. Modafinil, as dopamine re-uptake inhibitor, may prove useful in this condition $[5,15]$.

Modafinil is an organic compound that is an inhibitor of dopamine and norepinephrine re-uptake. It has a stimulating effect on central nervous activity, and it is employed in treating narcolepsy, shift work sleep disorder and sleep apnea, or is used as a stimulant [18]. Modafinil lacks narcotic potential, however, preliminary studies suggest that it may be addictive.

This drug exerts psychoactive and euphoric effects, as well as alters mood, thinking and perception. Both modafinil and its (R)-enantiomer - armodafinil - are under current pharmacotherapy investigation. These compounds have similar pharmaceutical activity and $\mathrm{pK}$ value, thus, the elimination coefficient is the only distinction between these two $[15,18]$. Modafinil is considered to be a promising, well tolerated medication, free of serious adverse effects [5]. Indeed, all the evidence indicate that it is an auspicious agent, which may primarily prove beneficial to patients with depressive episodes.

One of the trials undertaken to assess the effect of modafinil was designed for a group of 63 patients diagnosed with bipolar disease. During this study, the patients received their regular treatment. One group, however, received additional doses of modafinil, while the second was administered pramipexole (the efficacy of which was being investigated simultaneously). The trial pharmacotherapy lasted 12 weeks. After a comparison of both agents, it was revealed that modafinil exhibited much less acute side effects after 
12 weeks of administration, and far less chronic effects as assessed after 7-9 months. Even though the results are optimistic in terms of clinical symptoms, it is still stressed that further studies must be conducted - especially since the majority of patients were treatment-resistant [5].

Another study was of a greater patient-subject population size. In this trial, 257 individuals took part, all BD patients with major depressive episodes. Their pre-trial treatment included lithium, olanzapine and valproic acid, and, unfortunately, was ineffective. The trial was double-blind and placebo-controlled. The investigated substance was armodafinil, the enantiopure of the wakefulness-promoting agent. In the study, the drug revealed itself to be well-tolerated. Moreover, it significantly reduced or eliminated BD symptoms in a large number of the patients [15].

\section{TAMOXIFEN}

Tamoxifen is an organic chemical compound that is a selective estrogen receptor modulator (SERM). It is a synthetic drug with anti-estrogen properties, which is principally applied in breast cancer treatment, as well as in prevention of breast cancer in groups of high-risk female patients $[1,14]$. Tamoxifen binds with estrogen receptors inside tumor cells, which leads to the inhibition of growth factor synthesis and the stimulation of progesterone receptor formation. In addition, it targets estrogen receptors located within the skeletal system, thus effecting an increased bone density. However, tamoxifen exerts much more complicated effects within the central nervous system. Herein, it acts as an antagonist in one system, and as an agonist in the other [14].Tamoxifen had been observed to evoke depressive symptoms in patients treated for breast cancer. Thus, it has been perceived as being a potential medication in bipolar disease therapy. Due to this reason, it has also been assessed for its antimanic curative potential [14].

It is worth noting, that not so long ago, a consideration has been published concerning the impact of protein kinase $\mathrm{C}$ (PKC) on the pathophysiology and treatment of BD. PKC is comprised of a group of enzymes, largely present in the central nervous system, which have a significant role in the regulation of the properties exhibited by the pre- and postsynaptic neurotransmitters. Abundant evidence demonstrates that PKC activation implies an increase in levels of dopamine - a neurotransmitter associated with manic syndromes. What is more, a number of convergent preclinical studies provide evidence that chronic treatment with lithium and valproic acid in therapeutic doses may somehow influence the regulation of the PKC signal cascade. This gave rise to an investigation of the potential anti-manic properties of tamoxifen, which may act as PKC inhibitor, albeit only after being administered in proper doses. Due to its bloodbrain barrier penetration, the drug is, as well, effective in the treatment of malignant glioma, and is well-tolerated even in high doses [1].

Due to these discovered properties, further studies have been conducted to verify whether tamoxifen has therapeutic anti-manic potential. In one of such studies, tamoxifen and medroxyprogesterone (MPA) were investigated simultaneously. MPA is a steroidal progestin, a synthetic variant of the steroid hormone progesterone, and it is used as a contraceptive, in hormone replacement therapy, and for the treatment of endometriosis, as well as for several other indications. Both agents were used in a double-blind, placebo-controlled trial, in a group of 52 female patients, as an adjacent therapy to a traditional mood stabilizer. The treatment lasted 28 days. In comparison with a placebo, MPA administration showed that it can bring about a considerable reduction of manic symptoms. Nonetheless, tamoxifen also exhibited analogical effects, albeit to a lesser degree.

Another double-blind, placebo-controlled research project was dedicated to pharmacotherapy with sole administration of tamoxifen. Apart from the investigated drug, the patient-subjects received lithium as well. The study was comprised of 40 patients with current manic episodes. The trial lasted 6 weeks, and the effects were assessed by way of the YMRS scale. The study revealed a significant change between patients on tamoxifen and those on placebo. Indeed, the results are quite unequivocal: symptom reduction was obtained much faster in the patient-subjects receiving tamoxifen. What is more, only minor adverse effects were observed (some patients reported being slightly fatigued). Thus, this study demonstrates that the effects of treatment with both lithium and tamoxifen were superior to those achieved with lithium monotherapy, especially in that the adverse events are actually incomparable with the derived benefits.

The above described pharmaceuticals showed themselves to be effective in both replenishing and advancing therapeutic methods targeted at BD, apart from their regular function and standard application. Perhaps, these, by now, fairly well investigated substances will point to new pharmacotherapy directions and to new drug investigations. Hence, pharmacotherapy employing such new medications may be an alternative to the present conventional treatments, especially in drug-resistant mania [11].

\section{REFERENCES}

1. Amrollahi Z. et al.: Double-blind, randomized, placebo-controlled 6-week study on the efficacy and safety of the tamoxifen adjunctive to lithium in acute bipolar mania. J. Affect. Disord., 129, 327, 2011.

2. Anand A. et al.: Early antidepressant effect of memantine during augmentation of lamotrigine inadequate response in bipolar depression: a double-blind, randomized, placebo-controlled trial. Bipolar Disord., 14, 64, 2012.

3. Berk M. et al.: Maintenance $\mathrm{N}$-acetyl cysteine treatment for bipolar disorder: A double-blind randomized placebo controlled trial. Berk et al. BMC Med., 10, 91, 2012.

4. Berk M. et al.: The efficacy of $\mathrm{N}$-acetylcysteine as an adjunctive treatment in bipolar depression: An open label trial. J. Affect. Disord., 135, 389, 2011.

5. Dell'Osso B. et al.: Superior chronic tolerability of adjunctive modafinil compared to pramipexole in treatment-resistant bipolar disorder. J. Affect. Disord., 150, 130, 2013.

6. Fan A. et al.: Allopurinol augmentation in the outpatient treatment of bipolar mania: a pilot study. Bipolar Disord., 14, 206, 2012.

7. Gao Y. et al.: Memantine reduces mania-like symptoms in animal models. Psychiatry Res., 188, 366, 2011.

8. Ionescu D.F. et al.: A single infusion of ketamine improves depression scores in patients with anxious bipolar depression. Bipolar Disord., 17(4), 438, 2015, doi: 10.1111/bdi.12277. 
9. Jahangard L. et al.: In a double-blind, randomized and placebocontrolled trial, adjuvant allopurinol improved symptoms of mania in in-patients suffering from bipolar disorder; Eur. Neuropsychopharmacol., 24, 1210, 2014.

10. Koukopoulos A. et al.: The sustained mood-stabilizing effect of memantine in the management of treatment resistant bipolar disorders: Findings from a 12-month naturalistic trial; J. Affect. Disord., 136, 163, 2012.

11. Kulkarnia J. et al.: A four week randomised control trial of adjunctive medroxyprogesterone and tamoxifen in women with mania; Psychoneuroendocrino., 43, 52, 2014.

12. Lally N. et al.: Anti-anhedonic effect of ketamine and its neural correlates in treatment-resistant bipolar depression; Transl. Psychiatry, 4, e469; doi:10.1038/tp.2014.10, 2014.

13. Magalhaes P.V. et al.: $\mathrm{N}$-acetyl cysteine add-on treatment for bipolar II disorder: a subgroup analysis of a randomized placebo-controlled trial; J. Affect. Disord., 129, 317, 2011.

14. Nguyen T.V. et al.: Hormonal Treatments for Bipolar Disorder: A Review of the Literature; Journal of Behavioral and Brain Science, $2,48,2012$.
15. Niemegers P. et al.: Pharmacokinetic evaluation of armodafinil for the treatment of bipolar depression; Expert Opin. Drug Metab. Toxicol., 8(9), 1189, 2012.

16. Permoda-Osip A. et al: Factors connected with efficacy of single ketamine infusion in bipolar depression; Psychiatr. Pol., 48, 35, 2014.

17. Savitz J. et al:: Minocycline and aspirin in the treatment of bipolar depression: a protocol for a proof-of-concept, randomised, doubleblind, placebo-controlled, 2x2 clinical trial; BMJ Open, 2, e000643, 2011.

18. Sienaert P. et al.: Evidence-based treatment strategies for treatmentresistant bipolar depression: a systematic review; Bipolar Disord., $15,61,2013$.

19. Stanley I.: Lithium and the Other Mood Stabilizers Effective in Bipolar Disorder Target the Rat Brain Arachidonic Acid Cascade; ACS Chem. Neurosci., 5, 459, 2014.

20. Stertz L., Magalha P.V.S., Kapczinski F.: Is bipolar disorder an inflammatory condition? The relevance of microglial activation; Curr. Opin. Psychiatry, 26,19, 2013.

21. Weiser M. et al.: Allopurinol for mania: a randomized trial of allopurinol versus placebo as add-on treatment to mood stabilizers and/or antipsychotic agents in manic patients with bipolar disorder; Bipolar Disord., 16, 441, 2014. 